samples from 118 different tumour types and subtypes as well as 608 samples of 76 different normal tissue types was analysed. Villin positivity was found in 54 of tumour categories and was most commonly seen in adenocarcinomas and neuroendocrine tumours of the colorectum (60-100\%), upper gastrointestinal tract $(61-100 \%)$, bilio-pancreatic system $(25-86 \%)$, yolk sack tumours $(76 \%)$, and in mucinous carcinomas of the ovary (67\%). Villin was also common in various categories of extra-gastrointestinal neuroendocrine neoplasms $(22-41 \%)$, kidney tumours (up to $18 \%$ ), hepatocellular carcinoma (25\%), endometroid carcinoma of the ovary (20\%) and of the endometrium (12\%) as well as in adenocarcinomas of the lung $(12 \%)$. These data demonstrate that villin is frequently expressed in gastrointestinal, biliopancreatic, and neuroendocrine neoplasms, but at least weak villin positivity occurred in 54 different tumour categories. Villin immunohistochemistry should, thus, be applied as a part of a panel for the distinction of tumours.

\section{CARBOXYPEPTIDASE A1 (CPA1) IMMUNOHISTOCHEMISTRY IS HIGHLY SENSITIVE AND SPECIFIC FOR ACINAR CELL CARCINOMA (ACC) OF THE PANCREAS}

Ria Uhlig, Sören Weidemann, Doris Hoeflmayer, Sarah Minner, Guido Sauter, Ronald Simon, Till Clauditz, David Dum Institute of Pathology, University Medical Center HamburgEppendorf, Hamburg, Germany

Carboxypeptidase A1 (CPA1) is a zinc metalloprotease which is produced in pancreatic acinar cells and plays a role in cleaving C-terminal branched-chain and aromatic amino acids from dietary proteins. This study assessed the utility of immunohistochemical CPA1 staining for diagnosing pancreatic acinar cell carcinoma. A total of 15,680 tumour samples from 132 different tumour types and subtypes as well as 8 samples each of 76 different normal tissue types were analyzed by immunohistochemistry in a tissue microarray format. CPA1 was strongly expressed in acinar cells of all normal pancreas samples but not in any other normal tissues. CPA1 immunostaining was detected in $100 \%$ of 11 pancreatic acinar cell carcinomas and one mixed acinar endocrine carcinoma (MAEC), but absent in 449 pancreatic ductal adenocarcinomas, 75 adenocarcinomas of the ampulla Vateri, and 11,739 other evaluable cancers from 128 different tumour entities. In conclusion, our data show that CPA1 is a highly sensitive and largely specific marker for normal and neoplastic pancreatic acinar cells. CPA1 immunohistochemistry greatly facilitates the otherwise often difficult diagnosis of pancreatic acinar cell carcinoma.

\section{PATTERN OF PLACENTAL ALKALINE PHOSPHATASE (PLAP) EXPRESSION IN HUMAN TUMOURS: A TISSUE MICROARRAY STUDY ON 12,381 TUMOURS}

Viktor Reiswich, Andreas Luebke, Ria Uhlig,

Sören Weidemann, Katharina Möller, Guido Sauter,

Ronald Simon, Till Krech, David Dum

Institute of Pathology, University Medical Center Hamburg-

Eppendorf, Hamburg, Germany
Placental alkaline phosphatase (PLAP) is commonly expressed at high levels in testicular germ cell tumours. PLAP immunohistochemistry is thus often used to confirm this diagnosis, especially in case of putative metastases. However, other tumours can also express PLAP. In order to comprehensively determine PLAP expression in normal and tumour tissue, a tissue microarray containing 16,166 samples from 131 different tumour types and subtypes as well as 608 samples of 76 different normal tissue types was analysed by immunohistochemistry. A moderate to strong PLAP positivity was found in 27 (21\%) of 131 different tumour types including types with frequent positivity such as seminoma (96\%), embryonal carcinoma (85\%), and yolk sack tumours of the testis $(56 \%)$, but also tumour types with low frequent staining like oesophageal adenocarcinoma (10\%), invasive urothelial carcinoma (4\%), cholangiocarcinoma (2\%), and adenocarcinoma of the lung (1\%). In conclusion, our data identify several tumour entities that can show PLAP expression at comparable levels to testicular germ cell tumours. These tumour entities need to be considered in case of PLAP positive metastases. Low level PLAP expression can be found in various other tumour entities and should generally not be viewed as a strong argument for germ cell neoplasia.

\section{GATA3 EXPRESSION IN HUMAN TUMOURS: A TISSUE MICROARRAY STUDY ON 13,204 TUMOURS}

Anne Menz, Christoph Fraune, Guido Sauter, Ronald Simon, Ria Uhlig, Christian Bernreuther, Patrick Lebok, Sebastian Dwertmann Rico Institute of Pathology, University Medical Center HamburgEppendorf, Hamburg, Germany

GATA3 is a critical transcription factor for embryonic development of various tissues including breast and urothelium and is commonly used as a diagnostic marker for breast and urothelial cancer although it can also occur in other neoplasms. To comprehensively evaluate GATA3 expression tumour tissues, a tissue microarray containing 17,646 samples from 135 different tumour types was analysed by immunohistochemistry. GATA3 positivity was found in 69 different tumour types including 48 (35\%) with at least one moderately positive and 25 tumour types (18\%) with at least one strongly positive tumour. The highest positivity rates occurred in non-invasive papillary urothelial carcinoma (100\%), lobular carcinoma (98\%) and carcinoma of no special type of the breast ( $92 \%$ ), basal cell carcinoma of the skin $(97 \%)$, and invasive urothelial carcinoma $(73 \%)$. In breast cancer, low GATA3 staining was linked to high pT stage $(p=0.03)$, low BRE grade $(p<0.0001)$, HER2 overexpression $(p=0.0085)$, oestrogen and progesterone receptor negativity $(p<0.0001$ each) and reduced survival $(p=0.03)$. In summary, our data demonstrate that GATA3 positivity can occur in various tumour entities. Particularly high frequency and levels of GATA3 occur in breast and urothelial carcinoma. A reduced level of GATA3 reflects cancer progression and poor patient prognosis in these tumour entities.

\section{MUC5AC EXPRESSION IS LINKED TO MUCINOUS/ ENDOMETROID SUBTYPE AND ABSENCE OF NODAL METASTASIS IN OVARIAN CANCER}

